Research Paper:

\title{
The Role of Cognitive Emotion Regulation, Resilience and Sleep Disorders in Predicting Suicidal Ideation of Addicts Under Treatment
}

\author{
Matin Jahanchi ${ }^{1}$, Shahnam Abolghasemi ${ }^{1^{*}}$
}

1. Department of Psychology, Tonekabon Branch, Islamic Azad University, Tonekabon, Iran.

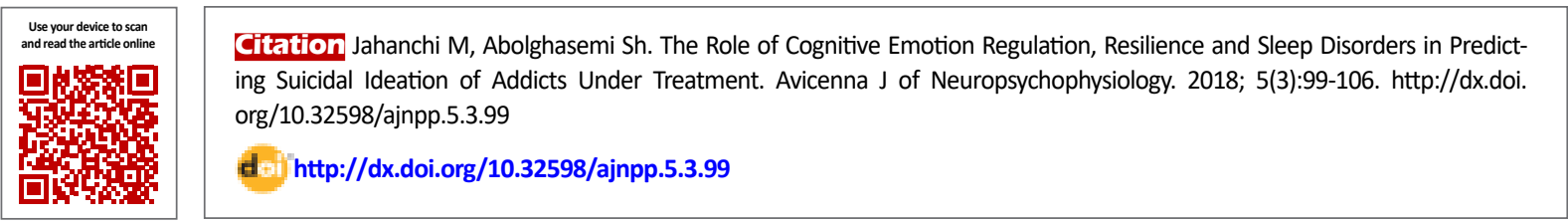

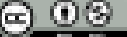

Article info:

Received: 07 Jan 2018

Accepted: 26 Jun 2018

Available Online: 01 Aug 2018
Keywords:

Suicidal ideation, Sleep-wake disorders, Emotions, Cognition

\section{A B S T RACT}

Introduction: Suicide is the most common cause of death among drug abusers, and drug abusers commit suicide ten times more than other people. Cognitive emotion regulation, resiliency and sleep disorder are effective on suicidal ideation

Objectives: The purpose of this study was to determine the role of cognitive emotion regulation, resiliency and sleep disorder in predicting suicidal ideation in addicts receiving treatment.

Materials and Methods: The statistical population of the study included all addicts under treatment, who referred to the addiction recovery centers of Rasht from September to December 2018. A total of 180 individuals were selected using the cluster sampling method. The following instruments were used in this research: the Suicide ideation Scale by Beck et al. (1979), the cognitive emotion regulation questionnaire by Garnefsky et al. (2001), Conner-Davidson resilience scale (2003), and Pittsburgh sleep quality index (1989). The Pearson correlation coefficient as well as multiple regression were used to analyze the collected data.

Results: The results showed a relationship between cognitive emotion regulation (positive and negative), resilience, and sleep disorders and suicidal ideation. According to the results, there was a negative and significant relationship between positive emotion regulation and resilience with suicidal ideation at the level of 0.01 , whereas there was a positive and significant relationship between the negative cognitive emotion regulation and sleep disorders, and suicidal ideation at the level of 0.01 . Sleep disorders, resilience, and negative cognitive emotion regulation were the best predictors of suicidal ideation and Sleep disorders, resilience, and negative cognitive emotion regulation at the level of 0.01 could explain about $20.7 \%$ of the variance of suicidal ideation.

Conclusion: It can be concluded that cognitive emotion regulation, resilience and sleep disorder can predict suicidal ideation of addicts under treatment. 


\section{Introduction}

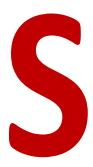

uicide is the most common cause of death among drug abusers. Drug abusers commit suicide ten times more than other people. Most addicts usually refer to treat addiction in the last phases of their problems, which makes him highly susceptible to suicidal ideation [1]. Other studies also have indicated that subjects committing suicide have a history of drug abuse and drug dependence [2]. Those with drug abuse are more likely to commit suicide [3]. In this regard, it is necessary to identify the factors influencing suicidal ideation in addicted people to prevent and reduce the risk of suicide in addicts.

Cognitive emotion regulation is one of the factors affective on suicidal ideation. It represents a range of processes, through which individuals can adjust the nature, fluctuation, and duration of emotions [4], which is appropriate for understanding emotional disorders. People suffering from emotional disorders tend to experience their own negative emotions as uncontrollable, and often lack the skills to manage and regulate these intense emotion experiences [5], which can increase physiological arousal and reduce self-control [6]. The manner, by which people deal with anger or regulate excitement, may be associated with suicidal behavior [6].

In general, individuals to deal with stressful experiences and situations, employ a variety of cognitive strategies, which are helpful in adjusting negative excitement and emotions in order to maintain their mental and emotional well-being [7]. It has also shown that there is a strong relationship between cognitive regulation strategies and emotional problems [8]. In investigating the relationship between strategies for cognitive regulation of emotion and emotional problems, researchers have found a significant positive correlation between emotional problems and maladaptive strategies of cognitive emotion regulation, and also a negative relationship between emotional problems and positive strategies of cognitive emotion regulation [9].

The level of individuals' resilience is another effective factor on suicidal ideation. It is defined as the ability to overcome difficulties and conquer the difficult conditions of life. Resilience includes situations leading to positive outcomes and succeeding in coping with challenges or stressful situations [10]. The best definition for resilience is to consider it as a successful adaptation to unfavorable conditions. The person's characteristics and position can determine resiliency processes, if such factors lead to healthy outcomes after stressful conditions. In other words, resilience is the person's ability in establishing a biological-physical balance in a difficult situation [11].

Sleep disorder has also shown effective on suicidal ideation. Several studies have confirmed the correlation between sleep disorder and quality and suicide [12]. The present research was designed to predict suicidal ideation and prevent and reduce suicide. It also aimed at finding an answer to the following question: Do cognitive emotion regulation, resiliency and sleep disorder can predict the suicidal ideation in addicts receiving treatment?

Suicide is one of the main causes of death among those with substance abuse and drug dependence disorders, and is also one of the main challenges in the treatment of these individuals. Studies on individuals committing suicide have shown that between $19 \%$ and $63 \%$ of them suffer from a type of substance-related disorder [13]. Consumption of drugs increases the likelihood of committing suicide, and substance abuse and dependence are one of the major risk factors of suicidal attempts $[14,15]$. The prevalence of suicidal ideation and suicidal behavior among addicts has been reported \%17 to $29 \%$ [16]. The purpose of this study was to determine the role of cognitive emotion regulation, resiliency and sleep disorder in predicting suicidal ideation in addicts under treatment.

\section{Methods}

The present research was a descriptive correlational study. Data collection was done by field method and using questionnaires. The statistical population included all addicts receiving treatment referring to the addiction recovery centers in Rasht from September to December 2012. Due to the lack of access to the exact number of the statistical population, the sample size was calculated based on the number of independent variables and for each variable 180 subjects were determined. Considering a possible drop in the sample size, 210 individuals were randomly selected through a random cluster sampling method.

After collecting the questionnaires, 30 questionnaires were excluded due to the lack of complete answers and 180 questionnaires were finally analyzed. For sampling, at first, 7 centers were randomly selected (Nedaye Slamat, Nasim Sahar, Forsat, Rahayesh, Moen, Nasr, and Raha) and 30 subjects were randomly selected from each center. It should be noted that the center's au- 
thorities made the subjects informed about the random selection of the samples. After referring to the addiction recovery centers and random selection, the questionnaires were delivered to the selected participants. Each questionnaire lasted $60 \mathrm{~min}$ to be filled. The written informed consent was obtained from the participants. Some questionnaires needed to be instructed by researcher.

\section{Beck suicide ideation scale}

The Beck Scale for Suicidal Ideation (BSSI) was designed by Beck et al. in 1979. It includs19 questions and each question has three options, which are scored from zero to two. Therefore, the total score is ranged from zero to 38 (depending on the presence or absence of risk of suicide and also different stages from thinking to committing suicide). BSSI has high reliability, with a Cronbach's alpha coefficient of 0.94 and also its reliability was obtained .74 using the test-retest method [17]. Ekramzadeh et al. [18] reported a simultaneous admissibility of the suicide ideation criterion of Beck with $\mathrm{GHQ}$ is equal to .95. The calculated permanency for this test is 0.80 , Alfa coefficient. Its reliability in the current research calculated .91 using Chronbach Alpha.

\section{Cognitive emotion regulation questionnaire}

The Cognitive Emotion Regulation Questionnaire (CERQ) was designed by Garnefski et al. (2001) as a multi-dimensional self-report instrument with 36 twoitem statements and has a special form for adults and children. CERQ evaluates 9 types of cognitive strategies, including self-blame, rumination, catastrophizing, other-blame, acceptance, positive refocusing, refocus on planning, putting into perspective, and positive reappraisal. It is a 36 -item scale, which is scored on fivepoint scale (from always to never) that every four questions evaluates one factor and in total, assesses the nine mentioned factors [19].

Garnefski et al. have reported an optimal validity and reliability for CERQ. According to Garnefski et al. [19], the Cronbach's alpha coefficient for the subscales of this questionnaire was ranged from 0.71 to 0.81 . Abdi [20] showed that the subscales of the Persian version of CERQ had a good internal consistency (a Cronbach's alpha of 0.76 to 0.92 ). The scores obtained from the items and also the total score of the subscales were significantly correlated ( $r=0.46$ to $r=0.75)$, and the obtained correlation coefficient ( 0.51 to 0.77 ) indicated its validity. In the current study, the validity and reliability of the CERQ were 0.73 and 0.79 , respectively.
Connor-Davidson Resilience Scale The Connor-Davidson Resilience Scale (CD-RISC) was developed by Connor and Davidson (2003) via a review on the studies on resiliency during 1979-1999. CD-RISC has 25 expressions that are scored on a Likert scale from 0 (completely false) to 4 (always true). Therefore, the maximum score of the CD-RISC is 100, and the score of each item is equal to the total score or the total values obtained from each items. The participant's level of resilience is the obtained score divided by 100 and multiplied by 100 .

A higher score (closer to 100), indicates high resilience, and a lower score (closer to 0 ), represents low resilience [21]. The correlation of each score with the total score, except for item 3, showed a coefficient of between 0.41 and 0.44 . The CD-RISC items were then analyzed by factor analysis method. Before factor extraction using the correlation matrix, the two KMO indices were 5556.28, each of which showed the adequacy of factor analysis. In addition, the validity and reliability of CD-RISC were 0.78 and 0.82 , respectively [22].

\section{Pittsburgh sleep quality index}

The Pittsburgh Sleep Quality Index (PSQI) is one of the best tools to measure sleep quality. PSQI was developed by Buysse et al. in 1989 at the Pittsburgh Psychiatric Institute. PSQI is a9-item scale (original scale), but from the 5th question, it contains 10 sub-items, therefore the entire questionnaire has 19 items, which are scored on a 4-point Likert scale from 0 to 3 . The questionnaire has 7 subscales as follows: Sleep mental quality, delay in sleeping, sleep duration, sleep efficiency, sleep disturbances, using sleeping medication, and daytime dysfunction.

To score the PSQI, three kinds of scores should be assigned to 19 items. Questions 1 and 3, questions 2 and 4 , and questions 5 to 9 are scored differently. Buysse et al. (1989) who developed and introduced the questionnaire for the first time reported internal consistency of 0.83 for the questionnaire using Cronbach's alpha. In its Persian version, the validity of the questionnaire was 0.86 and the reliability was 0.89 [23]. Also, in another study, the reliability of the PSQI using Cronbach's alpha and split-half methods was 0.46 and 0.52 , respectively [24]. Data were analyzed by SPSS V. 22 software using descriptive (Mean \pm SD) and also inferential statistics. To test the hypotheses of the research, the Pearson correlation test and multiple stepwise regression were used. 
Table 1. The Mean $\pm S D$ of the studied variables $(n=180)$

\begin{tabular}{cc}
\hline Variable & Mean \pm SD \\
\hline Positive emotion cognitive regulation & $74.40 \pm 3.89$ \\
Negative emotion cognitive regulation & $26.44 \pm 3.65$ \\
Resilience & $74.50 \pm 4.23$ \\
Sleep disorder & $17.79 \pm 3.96$ \\
Suicidal ideation & $31.84 \pm 3.77$ \\
\hline
\end{tabular}

AJNPP

Table 2. The correlation matrix of the cognitive emotion regulation (positive and negative), resiliency, sleep disorder, and suicidal ideation

\begin{tabular}{|c|c|c|c|c|c|}
\hline Variable & $\begin{array}{c}\text { Positive Cognitive Emo- } \\
\text { tion Regulation }\end{array}$ & $\begin{array}{l}\text { Negative Cognitive } \\
\text { Emotion Regulation }\end{array}$ & Resilience & $\begin{array}{l}\text { Sleep Disor- } \\
\text { der }\end{array}$ & $\begin{array}{l}\text { Suicidal } \\
\text { Ideation }\end{array}$ \\
\hline $\begin{array}{l}\text { Positive cognitive emotion } \\
\text { regulation }\end{array}$ & 1 & $-0.146^{*}$ & $0.458^{* *}$ & $-0.162^{* *}$ & $-0.265^{* *}$ \\
\hline $\begin{array}{l}\text { Negative cognitive emotion } \\
\text { regulation }\end{array}$ & - & 1 & $-0.174 * *$ & -0.095 & $0.264 * *$ \\
\hline Resilience & - & - & 1 & -0.231 & $-0.325^{* *}$ \\
\hline Sleep disorder & - & - & - & 1 & $0.357^{* *}$ \\
\hline Suicidal ideation & - & - & - & - & 1 \\
\hline
\end{tabular}

* $\mathrm{P}<0.05$;

AJNPP

$* * \mathrm{P}<0.01$

\section{Results}

The mean and standard deviation of the studied variables are indicated in Table 1. The results of Kolmogorov-Smirnov test showed the normal distribution of all variables, therefore, the parametric tests were used to test the research hypotheses using Pearson correlation coefficient.

According to Table 2, the results of the Pearson correlation coefficient indicated a linear relationship between emotion cognitive regulation (positive and negative), resiliency, sleep disorder, and suicidal ideation. There was a negative and significant correlation between positive emotion cognitive regulation and resiliency, and suicidal ideation at the level of 0.01 . There was also a positive and significant correlation between negative cognitive emotion regulation and sleep disorders, and suicidal ideation at the level of 0.01 .

To determine the best predictor of suicidal ideation among the variables, such as cognitive emotion regulation, resiliency and sleep disorders, a stepwise regression was used. It should be noted that the variables, including sleep disorders and cognitive emotion regulation were included in the model. The results are presented in Table 3 . This Table shows that sleep disorder could explain $12.2 \%$ of the variance of suicidal ideation $\left(\Delta R^{2}=0.122\right)$ and by adding resilience to the sleep disorder variable in the second model, a $6 \%$ increase was found in the variance in of suicidal ideation (0.181). Sleep disorders and resilience could explain $18.1 \%$ of the variance of suicidal ideation.

Table 3: Summary of the regression analysis of variables, including sleep disorders, resiliency, and negative cognitive emotion regulation

\begin{tabular}{cccccc}
\hline Variables of the Predictive Model & $\mathbf{R}$ & $\mathbf{R}^{\mathbf{2}}$ & $\mathbf{\Delta R}^{\mathbf{2}}$ & Standard Error \\
\hline Sleep disorders & 0.357 & 0.127 & 0.122 & 3.53 \\
Sleep disorders, resilience & 0.436 & 0.190 & 0.181 & 3.41 \\
Sleep disorders, resilience, negative cognitive emotion regulation & 0.470 & 0.221 & 0.207 & 3.36
\end{tabular}


Table 4. Analysis of variance to investigate the significance of sleep disorders, resilience, and negative cognitive emotion regulation

\begin{tabular}{|c|c|c|c|c|c|c|}
\hline Statistical Index & Sources of Changes & Sum of Squares & df & Mean of Squares & F-test & Sig. \\
\hline Regression & Sleep disorders & 324.121 & 1 & 324.121 & & \\
\hline Remaining & - & 2221.524 & 178 & 12.480 & 25.970 & 0.000 \\
\hline Total & - & 2545.644 & 179 & & & \\
\hline Regression & Sleep disorders and resilience & 482.972 & 2 & 241.486 & & \\
\hline Remaining & - & 2062.673 & 177 & 11.654 & 20.722 & 0.000 \\
\hline Total & - & 2545.644 & 179 & & & \\
\hline \multirow[t]{2}{*}{ Regression } & $\begin{array}{c}\text { Sleep disorders and resilience, } \\
\text { and negative cognitive emotion } \\
\text { regulation }\end{array}$ & 561.471 & 3 & 187.157 & & \\
\hline & & & & & 16.601 & 0.000 \\
\hline Remaining & - & 1984.174 & 176 & 11.274 & & \\
\hline Total & - & 2545.644 & 179 & & & \\
\hline
\end{tabular}

AJNPP

Adding resilience to the sleep disorder variable in the third model, resulted in a $2 \%$ increase in the variance of suicidal ideation $\left(\Delta R^{2}=0.207\right)$, and sleep disorders, resilience, and cognitive emotion regulation (negative) justified about $20.7 \%$ of the variance of suicidal ideation. Durbin-Watson statistic tests the assumptions of the residual errors. The values of between 1.5 and 2.5 show the absence of auto-correlation in the data. In this study, it obtained 1.757; therefore, a regression analysis was performed. Based on the Table 4, there was a correlation between sleep disorders, resilience, cognitive emotion regulation (negative) and suicide ideation. In addition, sleep disorders, resilience, and cognitive emotion regulation (negative) were predictors of the suicidal ideation.

\section{Discussion}

The aim of this research was to determine the role of cognitive emotion regulation, resilience and sleep disorders in predicting suicidal ideation in addicts receiving treatment. The results showed that there was a relationship between cognitive emotion regulation (positive and negative), resilience, sleep disorders, and suicidal ideation. Based on the findings, there was a negative correlation between cognitive emotion regulation (positive), resilience and suicidal ideation. There was also a positive relationship between cognitive emotion (negative), sleep disorders and suicidal ideation. In order to determine the best predictor of suicidal ideation among predictive variables, including cognitive emotion regulation, resilience and sleep disorders, and

Table 5. Stepwise regression analysis of the sleep disorders, resilience, and cognitive emotion regulation (negative)

\begin{tabular}{ccccccc}
\hline Variables & $\begin{array}{c}\text { Indices } \\
\text { Change Resources }\end{array}$ & Regression & $\begin{array}{c}\text { Standard } \\
\text { Error }\end{array}$ & $\begin{array}{c}\text { Standard } \\
\text { Regression }\end{array}$ & t-test & Sig. \\
\hline \multirow{2}{*}{ First model } & Fixed ratio & 25.800 & - & - & - & - \\
& Sleep disorders & 0.340 & 0.067 & 0.357 & 5.096 & 0.000 \\
\multirow{2}{*}{$\begin{array}{c}\text { Second } \\
\text { model }\end{array}$} & Fixed ratio & 32.540 & - & - & - & - \\
& Sleep disorders & 0.283 & 0.066 & 0.298 & 4.281 & 0.000 \\
& Resilience & -0.064 & 0.017 & -0.257 & -3.692 & 0.000 \\
& Fixed ratio & 26.305 & - & - & - & - \\
Third model & Sleep disorders & 0.273 & 0.065 & 0.302 & 4.422 & 0.000 \\
& Resilience & -0.056 & 0.017 & -0.228 & -0.3 .292 & 0.001 \\
& Negative cognitive motion & 0.185 & 0.070 & 0.179 & 2.639 & 0.009 \\
\hline & regulation & & & & & AJNPP
\end{tabular}


the stepwise regression was used and resilience, sleep disorders, and negative cognitive emotion regression included in the model.

Sleep disorders, resilience, and negative cognitive emotion regulation could predict about $20.7 \%$ of the variance of suicidal ideation. In general, the research hypothesis, according to which cognitive emotion regulation, resilience, and sleep disorders can predict suicidal ideation of the addicts under treatment, was confirmed. Sleep disorders and cognitive emotion regulation were the best predictors of suicidal ideation. The research results are consistent with the results of Richardson et al. [25], Cooley \& Fite [26], and Walker [27].

The individual and environmental factors can help an individual to reduce suicidal thoughts. Cognitive emotion regulation is one of the effective factors. Cognition and emotions are two important aspects of human life, and their interaction is obvious in the manifestation of most of the individuals' behaviors. Therefore, for a better understanding of the suicidal behaviors and their correlations with other behaviors, studying both cognitive and emotion constructs is essential, which is partially observed in the Schneiderman study [28].

In addition, contrary to the uncompromising strategies of cognitive emotion regulation, such as self-blaming and disaster, which are the core of emotional problems, compromised strategies, such as re-evaluation and acceptance in the field of psychology are currently proposed as the therapeutic techniques. Therefore, the cognitive ability to manage emotions makes individuals enable to use adaptive emotion strategies for suicidal ideation. Addicts face more difficulties in emotion regulation than others [29], and since the lack of excitement management can lead to the feelings, such as failure, hopelessness, low self-esteem, isolation, etc., these factors can be the causes of suicidal ideation. Therefore, it can be concluded that cognitive emotion regulation can predict suicide.

Based on the results, resilience is another factor to reduce suicidal ideation. It is preventive against mental disorders and a high level of resilience helps the individual to use positive feelings and emotions ending undesirable experiences and passing to the desired state. Resilience also provides the person an ability to make a more realistic and positive assessment of events, and can more logically manage his emotions. These people can choose more effective solutions to the problems and difficulties of life, and use less harmful and inefficient practices, such as ending his/her life.
Another result showed that sleep disorders are related to suicidal ideation and can predict suicide. Difficulty in sleeping is one of the important aspects of physical and psychological misconduct behaviors in drug abusers [26]. In the acute phase of drug abuse, an increase is seen in the delay in falling asleep and also the total sleeping time decreases. At this stage, the numbers of slow waves come back to normal levels, but the rate of sleep increases with rapid reactive or rebounded forms of eye movements.

On the other hand, emotional memory and fixation of the inputs that somehow accompany with the individual's sense of prosperity are completely done in REM sleep. In addition, the lack of using substances can increase the REM sleep density and emotions are at the higher risk of stabilization during sleep [27]. Therefore, sleep disorder that disturbs the rhythm of sleep can cause cognitive disorders in the individual. Due to the inability of the addict to control thoughts and impulses, this disorder can lead to suicidal ideation in the helpless individual.

In explaining this finding, it can be said that emotion regulation is considered as the process, by which emotions are adjusted to respond to the perceptible and non-existent environmental expectations [26]. Emotion regulation has three important features. First, it is likely that people will adjust their negative and positive emotions by both decreasing and increasing their emotions. [25]. This emotion regulation usually has a social nature and, although individuals do not regulate their positive emotions, they report these emotions less than negative emotions.

Secondly, the primary components of the emotion regulation are alerting and essentially happen deliberately and thoughtfully, but they then happen without consciousness and awareness. Third, it has no default, whether the regulation of emotion is necessarily good or bad [26]. In fact, the negative correlation of positive strategies with suicide is due to the reason that when one evaluates a negative event in his mind with these positive strategies, he/she looks at the event with a more positive attitude, and considers its possible short and long-term positive aspects. As a result, they experience a lower level of depression and disappointment and therefore are less susceptible to suicidal ideation. In general, when people with full awareness and cognition of their thoughts use positive strategies, they substitute positive thoughts for suicidal ideation by correct emotion recognition.

Thirty subjects were excluded from the study, due to their psychological and situational situations, which is one of the limitations of the present research. In addi- 
tion, using the self-report tool could affect the results. Considering the measures to encourage more people to participate in the research and using other data collection methods other than questionnaires, such as interviews and observations are suggested.

Regarding the results indicating a relationship between cognitive emotion regulation and suicidal thoughts, and as the cognitive emotion regulation could predict suicidal ideation, it is suggested that in order to prevent and reduce suicidal ideation, cognitive emotion regulation be considered along with other interventions for drug abusers. Resilience inversely affected suicide ideation, and it predicts the suicidal ideation as a criterion variable. It is suggested that, in order to prevent and reduce suicidal ideation, individuals can be empowered by appropriate training. According to the results of this study, there was a relationship between sleep disorders and suicidal ideation and also sleep disorders could predict suicidal ideation. Sleep disorders had a direct impact on suicidal ideation. Accordingly, in the treatment of addiction, the patient's problems should be more considered. It can be concluded that cognitive emotion regulation, resilience and sleep disorders can predict suicidal ideation of addicts receiving treatment.

\section{Ethical Considerations}

\section{Compliance with ethical guidelines}

All ethical principles were considered in this research. The participants were informed about the purpose of the research and its stages. The informed consent was obtained from the subjects. They were also assured of the confidentiality of their information. Moreover, the subjects were free to withdraw from the study if desired. They were also informed that they will be provided with the results of the research.

\section{Funding}

This research did not receive any specific grant from funding agencies in the public, commercial, or not-forprofit sectors.

\section{Authors' contributions}

All authors contributed equally in preparing all parts of the research.

\section{Conflict of interest}

The authors declared no conflict of interests.

\section{References}

[1] Askian P, Krauss SE, Baba M, Abdul Kadir R, Sharghi HM. Characteristics of co-dependence among wives of persons with substance use disorder in Iran. International Journal of Mental Health and Addiction. 2016; 14:268-83. [DOI:10.1007/s11469-016-9631-0]

[2] Dewald JF, Meijer AM, Oort FJ, Kerkhof GA, Bögels SM. The influence of sleep quality, sleep duration and sleepiness on school performance in children and adolescents: A meta-analytic review. Sleep Medicine Reviews. 2010; 14(3):179-89. [DOI:10.1016/j. smrv.2009.10.004] [PMID]

[3] Dragisic T, Dickov A, Dickov V, Mijatovic V. Drug addiction as risk for suicide attempts. Materia Sociomedica. 2015; 27(3):188-91. [DOI:10.5455/msm.2015.27.188-191] [PMID] [PMCID]

[4] Sakiris N, Berle D. A systematic review and meta-analysis of the Unified Protocol as a transdiagnostic emotion regulation based intervention. Clinical Psychology Review. 2019; 72:101751. [DOI:10.1016/j.cpr.2019.101751] [PMID]

[5] Garnefski N, Kraaij V, Spinhoven P. Negative life events, cognitive emotion regulation and emotional problems. Personality and Individual Differences. 2001; 30(8):1311-27. [DOI:10.1016/S01918869(00)00113-6]

[6] Segal B, Morral AR, Stevens SJ. Adolescent substance abuse treatment in the United States: Exemplary models from a national evaluation study. New York: Routledge; 2014 [DOI:10.4324/9781315821344]

[7] Nock MK, Borges G, Bromet EJ, Cha CB, Kessler RC, Lee S. Suicide and suicidal behavior. Epidemiologic Reviews. 2008; 30:133-54 [DOI:10.1093/epirev/mxn002] [PMID] [PMCID]

[8] Hanewald R. Reviewing the literature on "at-risk" and resilient children and young people. Australian Journal of Teacher Education. 2011; 36(2):16-29. [DOI:10.14221/ajte.2011v36n2.2]

[9] Neff KD, McGehee P. Self-compassion and psychological resilience among adolescents and young adults. Self and Identity. 2010; 9(3):225-40. [DOI:10.1080/15298860902979307]

[10] Kraaij V, van Emmerik A, Garnefski N, Schroevers MJ, Lo-Fo-Wong $D$, van Empelen P, et al. Effects of a cognitive behavioral self-help program and a computerized structured writing intervention on depressed mood for HIV-infected people: A pilot randomized controlled trial. Patient Education and Counseling. 2010; 80(2):200-4. [DOI:10.1016/j.pec.2009.08.014] [PMID]

[11] Le T, Guydish J, Pagano A, Tajima B, Passalacqua E. Smokers in addiction treatment see their risk of tobacco-related disease as lower than that of an average smoker. Drug and Alcohol Dependence. 2015; 146:e171. [DOI:10.1016/j.drugalcdep.2014.09.381]

[12] Legerstee JS, Garnefski N, Verhulst FC, Utens EM. Cognitive coping in anxiety-disordered adolescents. Journal of Adolescence. 2011; 34(2):319-26. [DOI:10.1016/j.adolescence.2010.04.008] [PMID]

[13] Maimon D, Browning CR, Brooks-Gunn J. Collective efficacy, family attachment, and urban adolescent suicide attempts. Journal of Health and Social Behavior. 2010; 51(3):307-24. [DOI:10.1177/0022146510377878] [PMID] [PMCID]

[14] Morris AS, Squeglia LM, Jacobus J, Silk JS. Adolescent brain development: Implications for understanding risk and resilience processes through neuroimaging research. Journal of Research on Adolescence. 2018; 28(1):4-9. [DOI:10.1111/jora.12379] [PMID] [PMCID] 
[15] Orford J, Velleman R, Natera G, Templeton L, Copello A. Addiction in the family is a major but neglected contributor to the global burden of adult ill-health. Social Science \& Medicine. 2013; 78:70-7. [DOI:10.1016/j.socscimed.2012.11.036] [PMID]

[16] Xia QR, Liang J, Cao Y, Shan F, Liu Y, Xu YY. Increased plasma nesfatin-1 levels may be associated with corticosterone, IL-6, and CRP levels in patients with major depressive disorder. Clinica Chimica Acta. 2018; 480:107-11. [DOI:10.1016/j.cca.2018.02.004] [PMID]

[17] Penney A, Mazmanian D, Jamieson J, Black N. Factors associated with recent suicide attempts in clients presenting for addiction treatment. International Journal of Mental Health and Addiction. 2012; 10:132-40. [DOI:10.1007/s11469-010-9307-0]

[18] Ekramzadeh S, Javadpour A, Draper B, Mani A, Withall A, Sahraian $A$. Prevalence and correlates of suicidal thought and selfdestructive behavior among an elderly hospital population in Iran. International Psychogeriatrics. 2012; 24(9):1402-8. [DOI:10.1017/ S1041610212000245] [PMID]

[19] Garnefski N, Kraaij V. Cognitive Emotion Regulation Questionnairedevelopment of a short 18-item version (CERQ-short). Personality and Individual Differences. 2006; 41(6):1045-53. [DOI:10.1016/j. paid.2006.04.010]

[20] Abdi S, Taban Sh, Ghaemian A. Cognitive Emotion Regulation Questionnaire: Validity and reliability of Persian translation of CERQ-36 item. Procedia - Social and Behavioral Sciences. 2012; 32:2-7. [DOI:10.1016/j.sbspro.2012.01.001]

[21] Roy A, Carli V, Sarchiapone M. Resilience mitigates the suicide risk associated with childhood trauma. Journal of Affective Disorders. 2011; 133(3):591-4. [DOI:10.1016/j.jad.2011.05.006] [PMID]

[22] Salo R, Gabay S, Fassbender C, Henik A. Distributed attentional deficits in chronic methamphetamine abusers: Evidence from the Attentional Network Task (ANT). Brain and Cognition. 2011; 77(3):446-52. [DOI:10.1016/j.bandc.2011.08.012] [PMID] [PMCID]

[23] Taylor DJ, Bramoweth AD. Patterns and consequences of inadequate sleep in college students: Substance use and motor vehicle accidents. The Journal of Adolescent Health. 2010; 46(6):610-2. [DOI:10.1016/j.jadohealth.2009.12.010] [PMID]

[24] Bender TW, Gordon KH, Bresin K, Joiner TE Jr. Impulsivity and suicidality: The mediating role of painful and provocative experiences. Journal of Affective Disorders. 2011; 129(1-3):301-7. [DOI:10.1016/j.jad.2010.07.023] [PMID]

[25] Glick M, Williams DM, Kleinman DV, Vujicic M, Watt RG, Weyant RJ. A new definition for oral health developed by the FDI World Dental Federation opens the door to a universal definition of oral health. British Dental Journal. 2016; 221(12):792-3. [DOI:10.1038/ sj.bdj.2016.953] [PMID]

[26] Cooley JL, Fite PJ. Peer victimization and forms of aggression during middle childhood: The role of emotion regulation. Journal of Abnormal Child Psychology. 2016; 44(3):535-46. [DOI:10.1007/ s10802-015-0051-6] [PMID]

[27] Walker MP. The role of sleep in cognition and emotion. Annals of the New York Academy of Sciences. 2009; 1156:168-97. [DOI:10.1111/j.1749-6632.2009.04416.x] [PMID]

[28] Schneider B, Kõlves K, Blettner M, Wetterling T, Schnabel A, Värnik $A$. Substance use disorders as risk factors for suicide in an Eastern and a Central European city (Tallinn and Frankfurt/Main). Psychiatry Research. 2009; 165(3):263-72. [DOI:10.1016/.jpsychres.2008.03.022] [PMID]
[29] Don Richardson J, King L, St Cyr K, Shnaider P, Roth ML, Ketcheson $F$, et al. Depression and the relationship between sleep disturbances, nightmares, and suicidal ideation in treatment-seeking Canadian Armed Forces members and veterans. BMC Psychiatry. 2018; 18(1):204. [DOI:10.1186/s12888-018-1782-z] [PMID] [PMCID] 